\title{
PULLBACK AND FORWARD ATTRACTORS FOR A DAMPED WAVE EQUATION WITH DELAYS
}

\author{
TOMÁS CARABALLO, PETER E. KLOEDEN, AND JOSÉ REAL
}

\begin{abstract}
The existence of a pullback (and also a uniform forward) attractor is proved for a damped wave equation containing a delay forcing term which, in particular, covers the models of Sine-Gordon type. The result follows from the existence of a compact set which is uniformly attracting for the two-parameter semigroup associated to the model.
\end{abstract}

\section{INTRODUCTION}

We aim to show in this paper how the theory of pullback attractors can be useful in the analysis of the asymptotic behaviour of damped wave equations subject to some kind of hereditary characteristics.

For partial differential equations with constant delay, the standard theory of global attractors can be successfully applied to handle this problem (see, Hale [9], Chueshov [6], etc ). However, when the hereditary characteristics are described by terms containing variable or distributed delays, the problem becomes nonautonomous. Although nonautonomous equations can be studied from several points of view (theory of skew product flows [16], kernel sections [5]), we will use the theory of pullback attractors for two-parameter semigroups or processes for several reasons. In particular, it allows us to obtain results for a wide class of delayed terms in a unified way without requiring compactness of the base space as in the skew product formalism or even the formulation of such a base space (see [4]).

In [3], it has been developed a program to prove that 2D-Navier-Stokes models with external forces containing bounded delays have pullback and forward attractors. The analysis there does not work in the present situation, as also happens in the non-delay case, since the hyperbolic structure of the equation only enables one to prove the existence of a compact attracting set instead of a compact absorbing set. This is not a consequence of the delay term, but rather of the hyperbolic nature of the equation (see [18]). The delay term considered here, however, is responsible of the nonautonomous character of the problem.

To show how the technique works, we will consider a model with a general functional term as an external force which, in particular, includes the models of Sine-Gordon type with delayed forcing term. Such equations, in which the nonlinearity is of the form $\sin u$, occur frequently in applications, and the technical steps needed to handle it are typical of other types of nonlinear terms (although it is complicated to handle a general nonlinearity which include them all). Since our primary aim is to investigate the effect of the delay, we restrict attention to globally Lipschitz nonlinearities like this representative one (see Section 3.4 for more details).

Date: June 8, 2004 . delay

Key words and phrases. Wave equations, pullback attractor, forward attractor, variable delay, distributed

(2000) Mathematics Subject Classification: 35R10, 35B40, 47H20, 58F39, 73K70.

Partly supported by Ministerio de Ciencia y Tecnología (Spain) and FEDER (European Community), projects HA2001-0075 and BFM2002-03068. 
In Section 2 we set our problem in a suitable nonautonomous framework, and recall some necessary concepts and results from the theory of pullback attractors. The existence of a pullback and a forward attractor is established in Section 3. Finally we include some technical but useful results in an Appendix.

\section{StATEMENT OF THE PROBLEM AND PRELIMinARIES}

2.1. The model. Let $\Omega \subset \mathbb{R}^{n}, n \geq 1$, be an open and bounded subset with smooth boundary $\partial \Omega=\Gamma$, and consider the following problem

$$
\left\{\begin{aligned}
\frac{\partial^{2} u}{\partial t^{2}}+\alpha \frac{\partial u}{\partial t}-\Delta u & =f+h\left(t, u_{t}\right), \quad t>\tau \\
u \mid \Gamma & =0, \quad t \geq \tau-r \\
u(x, t) & =\phi(x, t-\tau), \quad x \in \Omega, t \in[\tau-r, \tau], \\
\frac{\partial u}{\partial t}(x, t) & =\frac{\partial \phi}{\partial t}(x, t-\tau), \quad x \in \Omega, t \in[\tau-r, \tau],
\end{aligned}\right.
$$

where $f+h\left(t, u_{t}\right)$ is the source intensity which may depend on the history of the solution, $\alpha$ is a positive constant, $\phi$ is the initial datum on the interval $[\tau-r, \tau]$ where $r>0$, and $u_{t}$ is defined for $\theta \in[-r, 0]$ as $u_{t}(\theta)=u(t+\theta)$.

This problem can be set in an abstract framework by setting $H=L^{2}(\Omega)$ and $V=H_{0}^{1}(\Omega)$, which are Hilbert spaces for the usual inner products and associated norms given by

$$
\begin{array}{rlrl}
(u, v) & =\int_{\Omega} u(x) v(x) d x, & |u| & =\left(\int_{\Omega}|u(x)|^{2} d x\right)^{1 / 2}, \quad u, v \in H, \\
((u, v))=\sum_{i=1}^{n}\left(D_{i} u, D_{i} v\right), & \|u\| & =\left(\sum_{i=1}^{n} \int_{\Omega}\left|D_{i} u\right|^{2} d x\right)^{1 / 2}, \quad u, v \in V .
\end{array}
$$

Due to the continuous and dense injection $V \subset H$, identifying $H$ with its dual $H^{\prime}$, and denoting by $A: V \longrightarrow V^{\prime}$ the unbounded linear operator given by

$$
\langle A u, v\rangle=((u, v)), \quad u, v \in V,
$$

it follows that $A u=-\Delta u$ for any $u \in D(A)$, where $D(A)=\{u \in V: A u \in H\}=H_{0}^{1}(\Omega) \bigcap H^{2}(\Omega)$. We denote by $\lambda_{1}$ the first eigenvalue of $A$. Recall that $D(A)$ is also a Hilbert space for the norm $\|u\|_{D(A)}=|A u|, u \in D(A)$.

Our problem can be written as a second order differential equation in $H$ :

$$
\left\{\begin{aligned}
u^{\prime \prime}+\alpha u^{\prime}+A u & =f+h\left(t, u_{t}\right), \quad t>0, \\
u(t) & =\phi(t-\tau), \quad t \in[\tau-r, \tau], \\
u^{\prime}(t) & =\phi^{\prime}(t-\tau), \quad t \in[\tau-r, \tau] .
\end{aligned}\right.
$$

In general, if $\left(X,\|\cdot\|_{X}\right)$ is a Banach space, we denote by $C_{X}$ the space $C^{0}([-r, 0] ; X)$ with the sup-norm, i.e., $\|\phi\|_{C_{X}}=\sup _{\theta \in[-r, 0]}\|\phi(\theta)\|_{X}$, for $\phi \in C_{X}$.

Given another Banach space $\left(Y,\|\cdot\|_{Y}\right)$ such that the injection $X \subset Y$ is continuous, we denote by $C_{X, Y}$ the Banach space $C_{X} \cap C^{1}([-r, 0] ; Y)$ with the norm $\|\cdot\|_{C_{X, Y}}$ defined by

$$
\|\phi\|_{C_{X, Y}}^{2}=\|\phi\|_{C_{X}}^{2}+\left\|\phi^{\prime}\right\|_{C_{Y}}^{2}, \text { for } \phi \in C_{X, Y} .
$$

We will use the spaces $C_{D(A)}, C_{V}, C_{H}, C_{V, H}$, and $C_{D(A), V}$ in our analysis. We make the following hypotheses on the function $h: \mathbb{R} \times C_{H} \rightarrow H$.

(I) $\forall \xi \in C_{H}, t \in \mathbb{R} \rightarrow h(t, \xi) \in H$ is continuous,

(II) $\forall t \in \mathbb{R}, h(t, 0)=0$,

(III) $\exists L_{h}>0$ such that $\forall t \in \mathbb{R}, \quad \forall \xi, \eta \in C_{H}$

$$
|h(t, \xi)-h(t, \eta)| \leq L_{h}\|\xi-\eta\|_{C_{H}}
$$


(IV) $\exists m_{0} \geq 0, C_{h}>0$ such that $\forall m \in\left[0, m_{0}\right], \tau \leq t, u, v \in C^{0}([\tau-r, t] ; H)$

$$
\int_{\tau}^{t} \mathrm{e}^{m s}\left|h\left(s, u_{s}\right)-h\left(s, v_{s}\right)\right|^{2} \mathrm{~d} s \leq C_{h}^{2} \int_{\tau-r}^{t} \mathrm{e}^{m s}|u(s)-v(s)|^{2} \mathrm{~d} s .
$$

(V) $h \in C^{1}\left(\mathbb{R} \times C_{H} ; H\right)$, and there exists $C>0$ such that, for any $(t, \xi) \in \mathbb{R} \times C_{H}$ the Fréchet derivative $\delta h(t, \xi) \in \mathcal{L}\left(\mathbb{R} \times C_{H}, H\right)$ satisfies

$$
\|\delta h(t, \xi)\|_{\mathcal{L}\left(\mathbb{R} \times C_{H}, H\right)} \leq C\left(1+\|\xi\|_{C_{H}}\right) .
$$

Notice that condition (V) implies (I). However, we prefer to state both assumptions since some of our results hold true by assuming only conditions (I)-(IV).

We have the following result.

Theorem 2.1. Assume that $f \in L_{\text {loc }}^{2}(\mathbb{R}, H), \phi \in C_{V, H}$ and $h$ satisfies (I)-(IV). Then, for any $\tau \in \mathbb{R}$, there exists a unique solution $u(\cdot)=u(\cdot ; \tau, \phi)$ of the problem (2) such that $u \in$ $C^{0}([\tau-r, \infty) ; V) \cap C^{1}([\tau-r, \infty) ; H)$. If in addition $f^{\prime} \in L^{2}(\tau, T ; H)$ for all $T>0, \phi(0) \in C_{D(A)}$, $\phi^{\prime}(0) \in C_{V}$, then

$$
u \in C^{0}([\tau, \infty) ; D(A)) \cap C^{1}([\tau, \infty) ; V) .
$$

Proof. The existence is a consequence of a more general situation in [10] (see also [11] for a stochastic version). The regularity assertion follows from [14] (see also Appendix).

2.2. Preliminaries on pullback attractors. As the autonomous theory of attractors is not suitable to handle our model, we will use the theory of pullback attractors instead.

In the case of nonautonomous differential equations the initial time is just as important as the actual time, and the classical semigroup property of autonomous dynamical systems is no longer available. This is why the recent theory of pullback attractors has proven very useful, even for random dynamical systems (see [12], [13], [8]).

Instead of a family of one-parameter maps $S(t)$ we need to use a two-parameter semigroup or process $U(t, \tau)$ on the complete metric space $X$ (which in our case will be $C_{V, H}$ or $C_{D(A), V}$ ) (cf. Sell [16]); $U(t, \tau) \psi$ denotes the value of the solution at time $t$ which was equal to the initial value $\psi$ at time $\tau$.

The semigroup property is replaced by the process composition property

$$
U(t, \tau) U(\tau, r)=U(t, r) \quad \text { for all } \quad t \geq \tau \geq r
$$

and, obviously, the initial condition implies $U(\tau, \tau)=\mathrm{Id}$.

As in the standard theory of attractors, we seek an invariant attracting set. However, since the equation is nonautonomous this set also depends on time.

Definition 2.2. Let $U$ be a two-parameter semigroup on a complete metric space $X$. A family of compact sets $\{\mathcal{A}(t)\}_{t \in \mathbb{R}}$ is said to be a (global) pullback attractor for $U$ if, for all $\tau \in \mathbb{R}$, it satisfies

(i) $U(t, \tau) \mathcal{A}(\tau)=\mathcal{A}(t)$ for all $t \geq \tau$, and

(ii) $\lim _{s \rightarrow \infty} \operatorname{dist}_{X}(U(t, t-s) D, \mathcal{A}(t))=0$, for all bounded $D \subset X$, and all $t \in \mathbb{R}$.

The pullback attractor is said to be uniform if the attraction property is uniform in time, i.e.

$$
\lim _{s \rightarrow \infty} \sup _{t \in \mathbb{R}} \operatorname{dist}_{X}(U(t, t-s) D, \mathcal{A}(t))=0, \text { for all bounded } D \subset X .
$$

In the definitions, $\operatorname{dist}_{X}(A, B)$ is the Hausdorff semidistance between $A$ and $B$, defined as

$$
\operatorname{dist}_{X}(A, B)=\sup _{a \in A} \inf _{b \in B} d_{X}(a, b), \text { for } A, B \subseteq X .
$$

The notion of an attractor is closely related to those of pullback absorbing and attracting sets. 
Definition 2.3. The family $\{B(t)\}_{t \in \mathbb{R}}$ is said to be

(a) pullback absorbing with respect to the process $U$, if for all $t \in \mathbb{R}$ and all bounded $D \subset X$, there exists $T_{D}(t)>0$ such that for all $s \geq T_{D}(t)$

$$
U(t, t-s) D \subset B(t)
$$

(b) pullback attracting with respect to the process $U$, if for all $t \in \mathbb{R}$, all bounded $D \subset X$ and all $\varepsilon>0$, there exists $T_{\varepsilon, D}(t)>0$ such that for all $s \geq T_{\varepsilon, D}(t)$

$$
\operatorname{dist}_{X}(U(t, t-s) D, B(t))<\varepsilon ;
$$

(c) pullback uniformly absorbing (resp. uniformly attracting) if $T_{D}(t)$ in part (a) (resp. $T_{\varepsilon, D}(t)$ in part (b)) does not depend on the time $t$.

Related to these concepts we also have the notion of forward attracting sets.

Definition 2.4. A family of sets $\{B(t)\}_{t \in \mathbb{R}}$ is said to be forward attracting for $U$ if, for all $\tau \in \mathbb{R}$, it satisfies $\lim _{t \rightarrow \infty} \operatorname{dist}_{X}(U(t, \tau) D, B(t))=0$, for all bounded $D \subset X$.

The forward attraction is said to be uniform if

$$
\lim _{t \rightarrow \infty} \sup _{\tau \in \mathbb{R}} \operatorname{dist}_{X}(U(t+\tau, \tau) D, B(t+\tau))=0, \text { for all bounded } D \subset X .
$$

Remark 2.5. The previous definitions make also sense even if the family of operators $U(\cdot, \cdot)$ does not satisfy the properties of a two-parameter semigroup.

The existence of compact absorbing or attracting sets is crucial in order to prove the existence of pullback attractors. Due to the hyperbolicity of the equation, we will need the existence of compact attracting sets instead of absorbing ones. In the next theorem we collect some sufficient conditions ensuring these facts (see Crauel and Flandoli [7], Schmalfuss [15] or Crauel et al. [8]).

Theorem 2.6. Let $U(t, \tau)$ be a two-parameter process, and suppose $U(t, \tau): X \rightarrow X$ is continuous for all $t \geq \tau$. If there exists a family of compact pullback attracting sets $\{B(t)\}_{t \in \mathbb{R}}$, then there exists a pullback attractor $\{\mathcal{A}(t)\}_{t \in \mathbb{R}}$, such that $\mathcal{A}(t) \subset B(t)$ for all $t \in \mathbb{R}$, and which is given by

$$
\mathcal{A}(t)=\overline{\bigcup_{\substack{D \subset X \\ \text { bounded }}} \Lambda_{D}(t)} \text {, where } \Lambda_{D}(t)=\bigcap_{n \in \mathbb{N}} \overline{\bigcup_{s \geq n} U(t, t-s) D} .
$$

Remark 2.7. The uniqueness of the pullback attractor, as defined above, does not hold in general (see [1]). However, the one given in the preceding theorem is minimal with respect to set inclusion (see Crauel and Flandoli [7]). If we wish to guarantee uniqueness, we need to assume some additional hypotheses on the attractor, for instance, that the attractor belongs to a certain class of set valued functions (also called a universe), which are attracted by the attractor (see, e.g., Cheban et al. [4]). In particular, we may consider as a universe of attracted sets the family of uniformly bounded sets, which is equivalent to assume that the family $\{\mathcal{A}(t)\}_{t \in \mathbb{R}}$ is uniformly bounded (i.e there exists a bounded set $B \subset X$ such that $\mathcal{A}(t) \subset B$ for all $t \in \mathbb{R}$ ). A sufficient condition ensuring this is that the family of compact attracting sets in Theorem 2.6 is also uniformly bounded.

Remark 2.8. Notice that, when the attraction of $\{B(t)\}_{t \in \mathbb{R}}$ in Theorem 2.6 is uniform, the attractor need not be. On the other hand, if that family is given by a single set, i.e. $B(t)=B$, for all $t \in \mathbb{R}$, and the pullback attraction of this family is uniform, then this compact set $B$ is also uniformly forward attracting and, by means of the theory developed by Chepyzhov and Vishik [5], 
there exists a uniform forward attractor $\mathbb{A}_{f}$ which contains all the component sets of the pullback attractor. Moreover, it holds

$$
\bigcup_{t \in \mathbb{R}} \mathcal{A}(t) \subset \mathbb{A}_{f}
$$

being the inclusion strict in general (see Chepyzhov and Vishik [5] for more details).

The following result will be useful in order to prove the existence of the attracting family of sets.

Proposition 2.9. Let $U$ be a process in the normed linear space $X$, and suppose that $U$ can be written as

$$
U(t, \tau)=U_{1}(t, \tau)+U_{2}(t, \tau) \text { for all } t \geq \tau,
$$

and let $\{B(t)\}_{t \in \mathbb{R}}$ be a family of subsets of $X$ such that

$$
\begin{aligned}
& \lim _{s \rightarrow \infty} \operatorname{dist}_{X}\left(U_{1}(t, t-s) D, B(t)\right)=0, \quad \text { for all } t \in \mathbb{R}, \forall D \subset X \text { bounded } \\
& \text { (resp. } \left.\quad \lim _{s \rightarrow \infty} \sup _{t \in \mathbb{R}} \operatorname{dist}_{X}\left(U_{1}(t, t-s) D, B(t)\right)=0, \quad \forall D \subset X \quad \text { bounded }\right),
\end{aligned}
$$

and

$$
\begin{aligned}
& \lim _{s \rightarrow \infty}\left(\sup _{y \in D}\left\|U_{2}(t, t-s) y\right\|_{X}\right)=0, \quad \text { for all } t \in \mathbb{R}, \quad \forall D \subset X \text { bounded } \\
& \text { (resp. } \quad \lim _{s \rightarrow \infty} \sup _{t \in \mathbb{R}}\left(\sup _{y \in D}\left\|U_{2}(t, t-s) y\right\|_{X}\right)=0, \quad \forall D \subset X \quad \text { bounded). }
\end{aligned}
$$

Then,

$$
\begin{aligned}
& \lim _{s \rightarrow \infty} \operatorname{dist}_{X}(U(t, t-s) D, B(t))=0, \quad \text { for all } t \in \mathbb{R}, \forall D \subset X \text { bounded } \\
& \text { (resp. } \quad \lim _{s \rightarrow \infty} \sup _{t \in \mathbb{R}} \operatorname{dist}_{X}(U(t, t-s) D, B(t))=0, \quad \forall D \subset X \quad \text { bounded). }
\end{aligned}
$$

\section{EXISTENCE OF PULlBACK AND FORWARD ATTRACTORS}

We will proceed as follows. First, we will construct the evolution process associated to our problem. Then, we will prove the existence of a compact set which is uniformly attracting. As a consequence, the existence of a pullback attractor can be ensured as well as a forward uniform one.

3.1. The evolution process associated to the model. Consider our model (2) under the assumptions in Theorem 2.1 but, for simplicity, we assume that $f \in H$ (we will comment on the time dependent situation later on). We can construct a two-parameter semigroup or process $U(\cdot, \cdot)$ in $C_{V, H}$ as the family of mappings defined by $U(t, \tau)(\phi)=u_{t}(\cdot ; \tau, \phi) \quad \forall t \geq \tau, \phi \in C_{V, H}$. Observe that when $\phi \in C_{D(A), V}$, Theorem 2.1 implies that $u_{t}(\cdot ; \tau, \phi) \in C_{D(A), V}$, which means that we also have a process defined in the space $C_{D(A), V}$.

From now on we will argue only for the first case. The evolution property (3) for the family $\{U(t, \tau): t \geq \tau\}$ follows from the uniqueness of solutions established in Theorem 2.1. The continuity of each operator $U(t, \tau)$ is a consequence of the following result.

Lemma 3.1. Let $\phi, \psi \in C_{V, H}$ be two initial data for our problem (2), and let $\tau \in \mathbb{R}$ be an initial time. Denote by $u(\cdot)=u(\cdot ; \tau, \phi)$ and $v(\cdot)=u(\cdot ; \tau, \psi)$ the corresponding solutions to (2). Then, there exists a constant $\gamma>0$ which does not depend on the initial data and time, such that

$$
\|u(t)-v(t)\|^{2}+\left|u^{\prime}(t)-v^{\prime}(t)\right|^{2} \leq\left(1+\lambda_{1}^{-1} C_{h}^{2} r\right) e^{\gamma(t-\tau)}\|\phi-\psi\|_{C_{V, H}}^{2} \quad \forall t \geq \tau,
$$

and

$$
\left\|u_{t}-v_{t}\right\|_{C_{V, H}}^{2} \leq\left(1+\lambda_{1}^{-1} C_{h}^{2} r\right) e^{\gamma(t-\tau)}\|\phi-\psi\|_{C_{V, H}}^{2} \quad \forall t \geq \tau+r .
$$


Proof. Let us denote $w(\cdot)=u(\cdot)-v(\cdot)$. Then, we have

$$
\begin{aligned}
w^{\prime \prime}+\alpha w^{\prime}+A w & =h\left(t, u_{t}\right)-h\left(t, v_{t}\right), \\
\frac{1}{2} \frac{d}{d t}\left(|| w||^{2}+\left|w^{\prime}\right|^{2}\right)+\alpha\left|w^{\prime}\right|^{2} & =\left(h\left(t, u_{t}\right)-h\left(t, v_{t}\right), w^{\prime}\right) \\
& \leq\left|h\left(t, u_{t}\right)-h\left(t, v_{t}\right)\right|\left|w^{\prime}\right| \\
& \leq \frac{1}{2}\left|w^{\prime}\right|^{2}+\frac{1}{2}\left|h\left(t, u_{t}\right)-h\left(t, v_{t}\right)\right|^{2},
\end{aligned}
$$

and thus

$$
\frac{d}{d t}\left(|| w||^{2}+\left|w^{\prime}\right|^{2}\right) \leq\left|w^{\prime}\right|^{2}+\left|h\left(t, u_{t}\right)-h\left(t, v_{t}\right)\right|^{2} .
$$

Taking into account condition (IV)

$$
\begin{aligned}
\int_{\tau}^{t}\left|h\left(s, u_{s}\right)-h\left(s, v_{s}\right)\right|^{2} d s & \leq C_{h}^{2} \int_{\tau-r}^{t}|u(s)-v(s)|^{2} d s \\
& \leq \lambda_{1}^{-1} C_{h}^{2} r\|\phi-\psi\|_{C_{V}}^{2}+\lambda_{1}^{-1} C_{h}^{2} \int_{\tau}^{t}\|w(s)\|^{2} d s,
\end{aligned}
$$

from (6) we obtain by integrating over the interval $[\tau, t]$

$$
\begin{aligned}
\|w(t)\|^{2}+\left|w^{\prime}(t)\right|^{2} \leq & \|w(\tau)\|^{2}+\left|w^{\prime}(\tau)\right|^{2}+\lambda_{1}^{-1} C_{h}^{2} r\|\phi-\psi\|_{C_{V}}^{2} \\
& +\int_{\tau}^{t}\left(\lambda_{1}^{-1} C_{h}^{2}\|w(s)\|^{2}+\left|w^{\prime}(s)\right|^{2}\right) d s \\
\leq & \left(1+\lambda_{1}^{-1} C_{h}^{2} r\right)\|\phi-\psi\|_{C_{V, H}}^{2} \\
& +\int_{\tau}^{t}\left(\lambda_{1}^{-1} C_{h}^{2}\|w(s)\|^{2}+\left|w^{\prime}(s)\right|^{2}\right) d s,
\end{aligned}
$$

and, consequently,

$$
\|w(t)\|^{2}+\left|w^{\prime}(t)\right|^{2} \leq\left(1+\lambda_{1}^{-1} C_{h}^{2} r\right)\|\phi-\psi\|_{C_{V, H}}^{2}+\gamma \int_{\tau}^{t}\left(\|w(s)\|^{2}+\left|w^{\prime}(s)\right|^{2}\right) d s,
$$

where $\gamma=\max \left\{1, \lambda_{1}^{-1} C_{h}^{2}\right\}$. The Gronwall lemma implies now

$$
\|w(t)\|^{2}+\left|w^{\prime}(t)\right|^{2} \leq\left(1+\lambda_{1}^{-1} C_{h}^{2} r\right)\|\phi-\psi\|_{C_{V, H}}^{2} e^{\gamma(t-\tau)} \quad \text { for all } t \geq \tau .
$$

If we consider $t \geq \tau+r$, then $t+\theta \geq \tau$ for any $\theta \in[-r, 0]$ and

$$
\begin{aligned}
\|w(t+\theta)\|^{2}+\left|w^{\prime}(t+\theta)\right|^{2} & \leq\left(1+\lambda_{1}^{-1} C_{h}^{2} r\right)\|\phi-\psi\|_{C_{V, H}}^{2} e^{\gamma(t-\tau+\theta)} \\
& \leq\left(1+\lambda_{1}^{-1} C_{h}^{2} r\right)\|\phi-\psi\|_{C_{V, H}}^{2} e^{\gamma(t-\tau)} .
\end{aligned}
$$

Thus,

$$
\left\|w_{t}\right\|_{C_{V, H}}^{2} \leq\left(1+\lambda_{1}^{-1} C_{h}^{2} r\right)\|\phi-\psi\|_{C_{V, H}}^{2} e^{\gamma(t-\tau)}, \text { for } t \geq \tau+r
$$

Theorem 3.2. The mapping $U(t, \tau): C_{V, H} \rightarrow C_{V, H}$ is continuous for any $t \geq \tau$.

Proof. Let $\phi, \psi \in C_{V, H}$ be two initial data for our problem (2), and let $t \geq \tau$. Denote by $u(\cdot)=u(\cdot ; \tau, \phi)$ and $v(\cdot)=u(\cdot ; \tau, \psi)$ the corresponding solutions to (2). Then, writing again $w=u-v$ we obtain the following.

If $\tau-r \leq t \leq \tau$, then $w(t)=\phi(t-\tau)-\psi(t-\tau)$ and

$$
\begin{aligned}
\|w(t)\|^{2}+\left|w^{\prime}(t)\right|^{2} & \leq\|\phi-\psi\|_{C_{V}}^{2}+\left\|\phi^{\prime}-\psi^{\prime}\right\|_{C_{H}}^{2} \\
& \leq\left(1+\lambda_{1}^{-1} C_{h}^{2} r\right)\|\phi-\psi\|_{C_{V, H}}^{2} e^{\gamma(t-\tau+r)} .
\end{aligned}
$$


Thus we have

$$
\|w(t)\|^{2}+\left|w^{\prime}(t)\right|^{2} \leq\left(1+\lambda_{1}^{-1} C_{h}^{2} r\right)\|\phi-\psi\|_{C_{V, H}}^{2} e^{\gamma(t-\tau+r)} \text { for } t \geq \tau-r,
$$

whence

$$
\left\|w_{t}\right\|_{C_{V, H}}^{2} \leq\left(1+\lambda_{1}^{-1} C_{h}^{2} r\right)\|\phi-\psi\|_{C_{V, H}}^{2} e^{\gamma(t-\tau+r)} \text { for } t \geq \tau,
$$

which implies the continuity of $U(t, \tau)$ in its state variable.

3.2. Existence of bounded absorbing sets in $C_{V, H}$. We will consider in our computations an equivalent norm in the space $C_{V, H}$. For each $\varepsilon \in \mathbb{R}$, the norm $\|\phi\|_{\varepsilon}^{2}=\|\phi\|_{C_{V}}^{2}+\left\|\phi^{\prime}+\varepsilon \phi\right\|_{C_{H}}^{2}$, $\phi \in C_{V, H}$, is equivalent to $\|\cdot\|_{0}:=\|\cdot\|_{C_{V, H}}$.

This allows us to obtain absorbing balls for the original norm by proving the existence of absorbing balls for this new norm for some suitable value of $\varepsilon$. Indeed, let us denote

$$
B_{\varepsilon}(0, \rho)=\left\{\phi \in C_{V, H}:\|\phi\|_{\varepsilon}<\rho\right\} .
$$

Noticing that for $c_{1}=\max \left\{2,1+2 \varepsilon^{2} \lambda_{1}^{-1}\right\}$ it follows that

$$
\begin{aligned}
\|\phi\|_{C_{V, H}}^{2} & =\|\phi\|_{C_{V}}^{2}+\left\|\phi^{\prime}+\varepsilon \phi-\varepsilon \phi\right\|_{C_{H}}^{2} \\
& \leq\|\phi\|_{C_{V}}^{2}+2\left\|\phi^{\prime}+\varepsilon \phi\right\|_{C_{H}}^{2}+2 \varepsilon^{2}\|\phi\|_{C_{H}}^{2} \\
& \leq\left(1+2 \varepsilon^{2} \lambda_{1}^{-1}\right)\|\phi\|_{C_{V}}^{2}+2\left\|\phi^{\prime}+\varepsilon \phi\right\|_{C_{H}}^{2} \\
& \leq c_{1}\|\phi\|_{\varepsilon}^{2},
\end{aligned}
$$

we then have $B_{\varepsilon}(0, \rho) \subset B_{0}\left(0, c_{1}^{1 / 2} \rho\right)$.

Theorem 3.3. Assume that Conditions (I)-(IV) hold with $m_{0}>0$, and that $f \in H$. Suppose in addition that $2 \sqrt{2} \lambda_{1}^{-1 / 2} C_{h}<\min \left\{\frac{\alpha}{4}, \frac{\lambda_{1}}{2 \alpha}\right\}$. Then, there exists a family $\{B(t)\}_{t \in \mathbb{R}}$ of bounded sets in $C_{V, H}$ which is uniformly pullback (and forward) absorbing for the process $U(\cdot, \cdot)$. Moreover, $B(t)=B^{0}$ for all $t \in \mathbb{R}$, where $B^{0}$ is a bounded set in $C_{V, H}$.

Proof. We first choose $\varepsilon>0$ such that $2 \sqrt{2} \lambda_{1}^{-1 / 2} C_{h}<\varepsilon<\min \left\{\frac{\alpha}{4}, \frac{\lambda_{1}}{2 \alpha}\right\}$. Let $D \subset C_{V, H}$ be a bounded set, i.e. there exists $d>0$ such that for any $\phi \in D$ it holds

$$
\|\phi\|_{\varepsilon}^{2}=\|\phi\|_{C_{V}}^{2}+\left\|\phi^{\prime}+\varepsilon \phi\right\|_{C_{H}}^{2} \leq d^{2} .
$$

Now denote by $u(\cdot)=u(\cdot ; \tau, \phi)$ the solution of problem (2). Writing $v=u^{\prime}+\varepsilon u$ and taking into account equality (28) in the Appendix, we have

$$
\frac{1}{2} \frac{d}{d t}\left(|v|^{2}+\|u\|^{2}\right)+\varepsilon\|u\|^{2}+(\alpha-\varepsilon)|v|^{2}-\varepsilon(\alpha-\varepsilon)(u, v)=(f, v)+\left(h\left(t, u_{t}\right), v\right) .
$$

Now, for $\alpha_{1}=\frac{\varepsilon}{2}$, we obtain

$$
\begin{aligned}
\varepsilon\|u\|^{2}+(\alpha-\varepsilon)|v|^{2}-\varepsilon(\alpha-\varepsilon)(u, v) & \geq \varepsilon\|u\|^{2}+(\alpha-\varepsilon)|v|^{2}-\frac{\varepsilon(\alpha-\varepsilon)}{\sqrt{\lambda_{1}}}\|u|\| v| \\
& \geq \varepsilon\|u\|^{2}+\frac{3 \alpha}{4}|v|^{2}-\frac{\varepsilon \alpha}{\sqrt{\lambda_{1}}}\|u\||| v \mid \\
& \geq \varepsilon\|u\|^{2}+\frac{3 \alpha}{4}|v|^{2}-\frac{\varepsilon}{2}\|u\|^{2}-\frac{\varepsilon \alpha^{2}}{2 \lambda_{1}}|v|^{2} \\
& \geq \frac{\varepsilon}{2}\|u\|^{2}+\frac{\alpha}{2}|v|^{2} \\
& \geq \alpha_{1}\left(\|u\|^{2}+|v|^{2}\right)
\end{aligned}
$$


Thus,

$$
\begin{aligned}
\frac{d}{d t}\left(\|u\|^{2}+|v|^{2}\right)+2 \alpha_{1}\left(\|\left. u\right|^{2}+|v|^{2}\right) & \leq 2|f||v|+2\left|h\left(t, u_{t}\right)\right||v| \\
& \leq \frac{\alpha_{1}}{2}|v|^{2}+\frac{\alpha_{1}}{2}|v|^{2}+\frac{2}{\alpha_{1}}\left|h\left(t, u_{t}\right)\right|^{2}+\frac{2}{\alpha_{1}}|f|^{2} \\
& \leq \alpha_{1}|v|^{2}+\frac{2}{\alpha_{1}}\left|h\left(t, u_{t}\right)\right|^{2}+\frac{2}{\alpha_{1}}|f|^{2},
\end{aligned}
$$

whence

$$
\frac{d}{d t}\left(\|u\|^{2}+\|v\|^{2}\right)+\alpha_{1}\left(\|u\|^{2}+|v|^{2}\right) \leq \frac{2}{\alpha_{1}}|f|^{2}+\frac{2}{\alpha_{1}}\left|h\left(t, u_{t}\right)\right|^{2} .
$$

As our assumptions ensure that $-\alpha_{1}+\frac{2 \lambda_{1}^{-1} C_{h}^{2}}{\alpha_{1}}<0$, we can then choose $m \in\left(0, m_{0}\right)$ small enough such that $m-\alpha_{1}+\frac{2 \lambda_{1}^{-1} C_{h}^{2}}{\alpha_{1}}<0$. For this choice, we have

$$
\frac{d}{d t}\left[e^{m t}\left(\|u\|^{2}+|v|^{2}\right)\right]=m e^{m t}\left(|| u \|^{2}+|v|^{2}\right)+e^{m t} \frac{d}{d t}\left[\|u\|^{2}+|v|^{2}\right]
$$

and

$$
\frac{d}{d t}\left[e^{m t}\left(\|u\|^{2}+|v|^{2}\right)\right] \leq\left(m-\alpha_{1}\right) e^{m t}\left(\|u\|^{2}+|v|^{2}\right)+\frac{2 e^{m t}}{\alpha_{1}}|f|^{2}+\frac{2 e^{m t}}{\alpha_{1}}\left|h\left(t, u_{t}\right)\right|^{2} .
$$

By integrating over the interval $[\tau, t]$, we deduce

$$
\begin{aligned}
e^{m t}\left(\|u(t)\|^{2}+|v(t)|^{2}\right) \leq & e^{m \tau}\left(\|u(\tau)\|^{2}+|v(\tau)|^{2}\right)+\int_{\tau}^{t} \frac{2 e^{m s}}{\alpha_{1}}|f|^{2} d s \\
& +\left(m-\alpha_{1}\right) \int_{\tau}^{t} e^{m s}\left(\|u(s)\|^{2}+|v(s)|^{2}\right) d s \\
& +\int_{\tau}^{t} \frac{2 e^{m s}}{\alpha_{1}}\left|h\left(t, u_{t}\right)\right|^{2} d s \\
\leq & e^{m \tau}\left(\|u(\tau)\|^{2}+|v(\tau)|^{2}\right)+\frac{2}{\alpha_{1} m}|f|^{2}\left(e^{m t}-e^{m \tau}\right) \\
& +\left(m-\alpha_{1}\right) \int_{\tau}^{t} e^{m s}\left(\|u(s)\|^{2}+|v(s)|^{2}\right) d s \\
& +\frac{2 C_{h}^{2} \lambda_{1}^{-1}}{\alpha_{1}} \int_{\tau-r}^{t} e^{m s}|| u(s) \|^{2} d s \\
= & e^{m \tau}\left(\|u(\tau)\|^{2}+|v(\tau)|^{2}\right)+\frac{2}{\alpha_{1} m}|f|^{2}\left(e^{m t}-e^{m \tau}\right) \\
& +\left(m-\alpha_{1}\right) \int_{\tau}^{t} e^{m s}\left(\|u(s)\|^{2}+|v(s)|^{2}\right) d s \\
& +\frac{2 C_{h}^{2} \lambda_{1}^{-1}}{\alpha_{1}}\left[\int_{\tau-r}^{\tau} e^{m s}|| \phi(s)\left\|^{2} d s+\int_{\tau}^{t} e^{m s}\right\| u(s) \|^{2} d s\right] \\
\leq & e^{m \tau} d^{2}\left(1+\frac{2 \lambda_{1}^{-1} C_{h}^{2} r}{\alpha_{1}}\right)+\frac{2}{\alpha_{1} m}|f|^{2}\left(e^{m t}-e^{m \tau}\right) \\
& +\left(m-\alpha_{1}+\frac{2 \lambda_{1}^{-1} C_{h}^{2}}{\alpha_{1}}\right) \int_{\tau}^{t} e^{m s}\left(\|u(s)\|^{2}+|v(s)|^{2}\right) d s \\
\leq & e^{m \tau} d^{2}\left(1+\frac{2 \lambda_{1}^{-1} C_{h}^{2} r}{\alpha_{1}}\right)+\frac{2}{\alpha_{1} m}|f|^{2}\left(e^{m t}-e^{m \tau}\right),
\end{aligned}
$$

and this implies, for $t \geq \tau$,

$$
\begin{aligned}
\|u(t)\|^{2}+|v(t)|^{2} & \leq e^{m \tau} e^{-m t} d^{2}\left(1+\frac{2 \lambda_{1}^{-1} C_{h}^{2} r}{\alpha_{1}}\right)+\frac{2}{\alpha_{1} m}|f|^{2}\left(1-e^{m \tau} e^{-m t}\right) \\
& \leq \frac{2}{\alpha_{1} m}|f|^{2}+e^{m \tau} e^{-m t} d^{2}\left(1+\frac{2 \lambda_{1}^{-1} C_{h}^{2} r}{\alpha_{1}}\right) .
\end{aligned}
$$


If we denote

$$
\rho_{0}^{2}=\frac{4 c_{1}}{\alpha_{1} m}|f|^{2}, \quad \hat{\rho}_{0}^{2}=c_{1}\left(1+\frac{2 \lambda_{1}^{-1} C_{h}^{2} r}{\alpha_{1}}\right)
$$

then (7) yields that

$$
\|u(t ; \tau, \phi)\|^{2}+\left|u^{\prime}(t ; \tau, \phi)\right|^{2} \leq \frac{\rho_{0}^{2}}{2}+\hat{\rho}_{0}^{2} d^{2} e^{m(\tau-t)}, \quad \forall t \geq \tau,
$$

and, in particular,

$$
\|u(t ; \tau, \phi)\|^{2}+\left|u^{\prime}(t ; \tau, \phi)\right|^{2} \leq \frac{\rho_{0}^{2}}{2}+\hat{\rho}_{0}^{2} d^{2}, \quad \forall t \geq \tau .
$$

Moreover, as $u(t ; \tau, \phi)=\phi(t-\tau)$ and $u^{\prime}(t ; \tau, \phi)=\phi^{\prime}(t-\tau)$ for $t \in[\tau-r, \tau]$, then Eq. (9) holds true for $t \geq \tau-r$.

If we take now $t \geq \tau+r$, then for all $\theta \in[-r, 0]$ we have $t+\theta \geq \tau$ and so

$$
\|u(t+\theta ; \tau, \phi)\|^{2}+\left|u^{\prime}(t+\theta ; \tau, \phi)\right|^{2} \leq \frac{\rho_{0}^{2}}{2}+\hat{\rho}_{0}^{2} d^{2} e^{m r} e^{m(\tau-t)},
$$

or, in other words,

$$
\|U(t, \tau) \phi\|_{C_{V, H}}^{2} \leq \frac{\rho_{0}^{2}}{2}+\hat{\rho}_{0}^{2} d^{2} e^{m r} e^{m(\tau-t)}, \quad \forall t \geq \tau+r, \phi \in D .
$$

Therefore, there exists $T_{D} \geq r$ such that

$$
\|U(t, t-s) \phi\|_{C_{V, H}}^{2} \leq \rho_{0}^{2}, \quad \forall t \in \mathbb{R}, s \geq T_{D}, \phi \in D,
$$

which means that the ball $B_{C_{V, H}}\left(0, \rho_{0}\right)=B^{0} \subset C_{V, H}$ is uniformly pullback absorbing for the process $U(\cdot, \cdot)$ (but it is not compact yet).

Remark 3.4. On the one hand, observe that if $t_{0} \in \mathbb{R}$ and $t \geq t_{0}$, then $u\left(t+\theta ; t_{0}-s, \phi\right)=$ $u\left(t+\theta ; t-\left(s+t-t_{0}\right), \phi\right)$ and $u^{\prime}\left(t+\theta ; t_{0}-s, \phi\right)=u^{\prime}\left(t+\theta ; t-\left(s+t-t_{0}\right), \phi\right)$ with $s+t-t_{0} \geq s$. As a consequence of (12) we have

$$
\left\|U\left(t, t_{0}-s\right) \phi\right\|_{C_{V, H}}^{2} \leq \rho_{0}^{2}, \quad \forall t_{0} \in \mathbb{R}, t \geq t_{0}, s \geq T_{D}, \phi \in D,
$$

or equivalently, we have $\forall t_{0} \in \mathbb{R}, t \geq t_{0}, \theta \in[-r, 0], s \geq T_{D}, \phi \in D$,

$$
\left\|u\left(t+\theta ; t_{0}-s, \phi\right)\right\|^{2}+\left|u^{\prime}\left(t+\theta ; t_{0}-s, \phi\right)\right|^{2} \leq \rho_{0}^{2} .
$$

On the other hand, Eq. (9) implies, $\forall t_{0} \in \mathbb{R}, s \in \mathbb{R}, t \geq t_{0}-s-r, \phi \in D$,

$$
\left\|u\left(t ; t_{0}-s, \phi\right)\right\|^{2}+\left|u^{\prime}\left(t ; t_{0}-s, \phi\right)\right|^{2} \leq \frac{\rho_{0}^{2}}{2}+\hat{\rho}_{0}^{2} d^{2} .
$$

3.3. Existence of attracting sets in $C_{V, H}$. Now we can prove the following result.

Theorem 3.5. In addition to the assumptions in Theorem 3.3, suppose that Condition (V) holds. Then, there exists a compact set $B^{2} \subset C_{V, H}$ which is uniformly pullback attracting for the process $U(\cdot, \cdot)$, and consequently, there exists the pullback attractor $\{\mathcal{A}(t)\}_{t \in \mathbb{R}}$, and the uniform forward one (in the sense of Chepyzhov and Vishik) $\mathbb{A}_{f} \subset C_{V, H}$. Moreover, $\mathcal{A}(t) \subset C_{D(A), V}$ for all $t \in \mathbb{R}$.

Proof. Let us fix $\varepsilon$ as in the proof of Theorem 3.3. Let $D \subset C_{V, H}$ be a bounded set, i.e. there exists $d>0$ such that for any $\phi \in D$ it holds $\|\phi\|_{\varepsilon}^{2} \leq d^{2}$, and so, $\|\phi\|_{C_{V, H}}^{2} \leq c_{1} d^{2}$. 
Denote, as usual, by $u(\cdot)=u(\cdot ; \tau, \phi)$ the solution of problem $(2)$, and consider the following problems.

$$
\begin{aligned}
& \left\{\begin{array}{l}
v^{\prime \prime}+\alpha v^{\prime}+A v=f+h\left(t, u_{t}\right), \quad t \geq \tau, \\
v(t)=0, \quad t \in[\tau-r, \tau], \\
v^{\prime}(t)=0, \quad t \in[\tau-r, \tau],
\end{array}\right. \\
& \left\{\begin{array}{l}
w^{\prime \prime}+\alpha w^{\prime}+A w=0, \quad t \geq \tau, \\
w(t)=\phi(t-\tau), \quad t \in[\tau-r, \tau], \\
w^{\prime}(t)=\phi^{\prime}(t-\tau), \quad t \in[\tau-r, \tau] .
\end{array}\right.
\end{aligned}
$$

From the uniqueness of solutions of problems (2), (15) and (16) it follows that

$$
u(\cdot)=v(\cdot)+w(\cdot), \text { for all } \tau \in \mathbb{R}, \text { and all } t \geq \tau-r .
$$

Consequently, $U(t, \tau)$ can be written as

$$
U(t, \tau)(\phi)=U_{1}(t, \tau)(\phi)+U_{2}(t, \tau)(\phi), \quad \forall \phi \in C_{V, H}, t \geq \tau-r .
$$

where $U_{1}(t, \tau)(\phi)=v_{t}(\cdot)=v_{t}(\cdot ; \tau, \phi)$ and $U_{2}(t, \tau)(\phi)=w_{t}(\cdot)=w_{t}(\cdot ; \tau, \phi)$ are the solutions of (15) and (16) respectively.

First, thanks to (9), but with $f=h=0$, it follows

$$
\|w(t ; \tau, \phi)\|^{2}+\left|w^{\prime}(t ; \tau, \phi)\right|^{2} \leq c_{1} d^{2}, \quad \forall t \geq \tau, \phi \in D,
$$

and, by means of (10),

$$
\left\|w_{t}(\cdot ; \tau, \phi)\right\|_{C_{V}}^{2}+\left\|w_{t}^{\prime}(\cdot ; \tau, \phi)\right\|_{C_{H}}^{2} \leq c_{1} d^{2} e^{m r} e^{m(\tau-t)}, \quad \forall t \geq \tau+r, \phi \in D .
$$

Furthermore, for $t_{0} \in \mathbb{R}, t \geq t_{0}$ and $s \geq T_{D} \geq r$,

$$
w\left(t ; t_{0}-s, \phi\right)=w\left(t ; t-\left(s+t-t_{0}\right), \phi\right)
$$

with $s+t-t_{0} \geq s \geq T_{D} \geq r$. Thus, Eq. (18) implies in particular

$$
\begin{aligned}
\left\|w\left(t ; t_{0}-s, \phi\right)\right\|^{2} & \leq c_{1} d^{2} e^{m r} e^{m\left(t_{0}-s-t\right)} \\
& \leq c_{1} d^{2} e^{m r} e^{-m s}, \forall t_{0} \in \mathbb{R}, t \geq t_{0}, s \geq T_{D}, \phi \in D .
\end{aligned}
$$

Then, (18) yields that

$$
\left\|U_{2}(t, t-s) \phi\right\|_{C_{V, H}}^{2} \leq c_{1} d^{2} e^{m r} e^{-m s}, \forall t \in \mathbb{R}, s \geq r, \phi \in D,
$$

whence

$$
\lim _{s \rightarrow+\infty} \sup _{t \in \mathbb{R}} \sup _{\phi \in D}\left\|U_{2}(t, t-s) \phi\right\|_{C_{V, H}}^{2}=0 .
$$

Let us now proceed with the other term. Let us fix $t_{0} \in \mathbb{R}, s \geq T_{D}, \phi \in D$ and denote

$$
u(t)=u\left(t ; t_{0}-s, \phi\right), v(t)=v\left(t ; t_{0}-s, \phi\right), \text { for } t \geq t_{0}-s-r,
$$

and

$$
F(t)=f+h\left(t, u_{t}\right), \text { for } t \geq t_{0}-s .
$$

Then,

$$
|F(t)| \leq|f|+L_{h}|| u_{t} \|_{C_{H}}
$$

From (13) we obtain

$$
|F(t)| \leq|f|+L_{h} \lambda_{1}^{-1 / 2} \rho_{0}=K_{1}, \quad \forall t \geq t_{0},
$$


and, from (14),

$$
\begin{aligned}
|F(t)| & \leq|f|+L_{h} \lambda_{1}^{-1 / 2}\left(\frac{\rho_{0}^{2}}{2}+\hat{\rho}_{0}^{2} d^{2}\right)^{1 / 2} \\
& \leq K_{1}+L_{h} \lambda_{1}^{-1 / 2} \hat{\rho}_{0} d, \quad \forall t \geq t_{0}-s .
\end{aligned}
$$

Next, we deduce from the assumptions on $h$ that

$$
F^{\prime}(t)=\left\langle\delta h\left(t, u_{t}\right),\left(1, u_{t}^{\prime}\right)\right\rangle,
$$

and

$$
\left|F^{\prime}(t)\right| \leq C\left(1+\left\|u_{t}\right\|_{C_{H}}\right)\left(1+\left\|u_{t}^{\prime}\right\|_{C_{H}}\right) .
$$

Arguing as we did in order to obtain (21) and (22), we have from (13) and (14)

$$
\left|F^{\prime}(t)\right| \leq C\left(1+\lambda_{1}^{-1 / 2} \rho_{0}\right)\left(1+\rho_{0}\right)=K_{2}, \quad \forall t \geq t_{0},
$$

and

$$
\begin{aligned}
\left|F^{\prime}(t)\right| & \leq C\left(1+\lambda_{1}^{-1 / 2}\left(\frac{\rho_{0}^{2}}{2}+\hat{\rho}_{0}^{2} d^{2}\right)^{1 / 2}\right)\left(1+\left(\frac{\rho_{0}^{2}}{2}+\hat{\rho}_{0}^{2} d^{2}\right)^{1 / 2}\right) \\
& =K_{3}(d), \quad \forall t \geq t_{0}-s .
\end{aligned}
$$

Let us denote $y(t)=\left\|v^{\prime}(t)+\frac{\alpha}{2} v(t)\right\|^{2}+|A v(t)-F(t)|^{2}$ and make use of the estimates in Theorem 3.9. On the one hand, for all $t \geq t_{0}-s$,

$$
\begin{aligned}
\frac{d}{d t}(y(t))+\frac{\alpha}{2} y(t) & \leq \alpha|F(t)|^{2}+\frac{4}{\alpha}\left|F^{\prime}(t)\right|^{2}+\frac{\alpha^{3}}{8}\|v(t)\|^{2} \\
& \leq \alpha\left(K_{1}+L_{h} \lambda_{1}^{-1 / 2} \hat{\rho}_{0} d\right)^{2}+\frac{4}{\alpha} K_{3}(d)^{2}+\frac{\alpha^{3}}{8}\|v(t)\|^{2}
\end{aligned}
$$

but, as (14) and (19) ensure

$$
\|v(t)\|^{2} \leq 2\|u(t)\|^{2}+2\|w(t)\|^{2} \leq \rho_{0}^{2}+2 \hat{\rho}_{0}^{2} d^{2}+2 c_{1} d^{2},
$$

if we denote by

$$
K_{4}(d)=\alpha\left(K_{1}+L_{h} \lambda_{1}^{-1 / 2} \hat{\rho}_{0} d\right)^{2}+\frac{4}{\alpha} K_{3}(d)^{2}+\frac{\alpha^{3}}{8}\left(\rho_{0}^{2}+2 \hat{\rho}_{0}^{2} d^{2}+2 c_{1} d^{2}\right),
$$

then, in particular,

$$
y^{\prime}(t)+\frac{\alpha}{2} y(t) \leq K_{4}(d), \quad \forall t \in\left[t_{0}-s, t_{0}\right] .
$$

Noticing that $y\left(t_{0}-s\right)=\left|F\left(t_{0}-s\right)\right|^{2}$, the Gronwall lemma leads us to

$$
y\left(t_{0}\right) \leq \frac{2}{\alpha} K_{4}(d)+\left(K_{1}+L_{h} \lambda_{1}^{-1 / 2} \hat{\rho}_{0} d\right)^{2}=K_{5}(d) .
$$

On the other hand, if $t \geq t_{0}$, we deduce from (13) and (19)

$$
\|v(t)\|^{2} \leq 2\|u(t)\|^{2}+2\|w(t)\|^{2} \leq 2 \rho_{0}^{2}+2 c_{1} d^{2 e^{m r} e^{-m s}},
$$

and, from (21) and (23)

$$
\begin{aligned}
y^{\prime}(t)+\frac{\alpha}{2} y(t) & \leq \alpha K_{1}+\frac{4}{\alpha} K_{2}+\frac{\alpha^{3}}{8}\|v(t)\|^{2} \\
& \leq \alpha K_{1}+\frac{4}{\alpha} K_{2}+\frac{\alpha^{3}}{4} \rho_{0}^{2}+\frac{\alpha^{3}}{4} c_{1} d^{2 e^{m r} e^{-m s}} \\
& =K_{6}+K_{7} d^{2} e^{-m s}, \quad \forall t \geq t_{0} .
\end{aligned}
$$


Once again, the Gronwall lemma implies that

$$
\begin{aligned}
y(t) & \leq y\left(t_{0}\right) e^{(\alpha / 2)\left(t_{0}-t\right)}+\frac{2}{\alpha} K_{6}+\frac{2}{\alpha} K_{7} d^{2} e^{-m s} \\
& \leq K_{5}(d) e^{(\alpha / 2)\left(t_{0}-t\right)}+\frac{2}{\alpha} K_{6}+\frac{2}{\alpha} K_{7} d^{2} e^{-m s}, \quad \forall t \geq t_{0} .
\end{aligned}
$$

Then, there exists $T_{D}^{\prime} \geq T_{D}$ such that, if $s \geq T_{D}^{\prime}$,

$$
y(t) \leq K_{5}(d) e^{(\alpha / 2)\left(t_{0}-t\right)}+\frac{3}{\alpha} K_{6}, \quad \forall t_{0} \in \mathbb{R}, t \geq t_{0} .
$$

Recalling that $y(t)=y\left(t ; t_{0}-s, \phi\right)$, if we fix $t \geq t_{0}$, take $s=T_{D}^{\prime}$ and denote $\tilde{s}=t-t_{0}+T_{D}^{\prime}$ we have, provided $t-t_{0}$ is large enough, that

$$
y\left(t ; t_{0}-T_{D}^{\prime}, \phi\right)=y\left(t ; t-\left(t-t_{0}+T_{D}^{\prime}\right), \phi\right)=y(t ; t-\tilde{s}, \phi) \leq \frac{4}{\alpha} K_{6} .
$$

In conclusion, there exists $T_{D}^{\prime \prime}>0$ such that for all $t \in \mathbb{R}$, and all $s \geq T_{D}^{\prime}+T_{D}^{\prime \prime}$,

$$
y(t ; t-s, \phi) \leq \frac{4}{\alpha} K_{6}, \quad \forall \phi \in D .
$$

Denoting $\hat{T}_{D}=T_{D}^{\prime}+T_{D}^{\prime \prime}+r$, we have for all $\phi \in D, t \in \mathbb{R}, s \geq \hat{T}_{D}$

$$
\left\|v^{\prime}(t ; t-s, \phi)+\frac{\alpha}{2} v(t ; t-s, \phi)\right\|^{2}+|A v(t ; t-s, \phi)-F(t ; t-s, \phi)|^{2} \leq \frac{4}{\alpha} K_{6},
$$

where $F(t ; t-s, \phi)=f+h\left(t, u_{t}(\cdot ; t-s, \phi)\right)$.

But, as for all $\phi \in D, t \in \mathbb{R}$ and $s \geq T_{D}$ it follows from (12) that $\|v(t ; t-s, \phi)\|^{2} \leq \rho_{0}^{2}$ and

$$
|F(t ; t-s, \phi)|^{2} \leq K_{7}^{2}=2|f|^{2}+2 L_{h}^{2} \lambda_{1}^{-1} \rho_{0}^{2},
$$

and, consequently, for all $\phi \in D, t \in \mathbb{R}, s \geq \hat{T}_{D}$,

$$
\left\|v^{\prime}(t ; t-s, \phi)\right\|^{2}+|A v(t ; t-s, \phi)|^{2} \leq \frac{8}{\alpha} K_{6}+\frac{\alpha^{2}}{2} \rho_{0}^{2}+4|f|^{2}+4 L_{h}^{2} \lambda_{1}^{-1} \rho_{0}^{2},
$$

and, by repeating once more the same argument previously used,

$$
\left\|v_{t}(\cdot ; t-s, \phi)\right\|_{C_{D(A), V}}^{2} \leq \rho_{1}^{2}=\frac{8}{\alpha} K_{6}+\frac{\alpha^{2}}{2} \rho_{0}^{2}+4|f|^{2}+4 L_{h}^{2} \lambda_{1}^{-1} \rho_{0}^{2},
$$

for all $\phi \in D, t \in \mathbb{R}, s \geq \hat{T}_{D}$.

This means that the ball $B^{1}=B_{C_{D(A), V}}\left(0, \rho_{1}\right)$ is a bounded set in $C_{D(A), V}$ which, in addition, is uniformly pullback absorbing for the family of operators $U_{1}(\cdot, \cdot)$. As $B^{1}$ is a bounded set in $C_{V, H}$, then there exists $T_{B^{1}} \geq r$ such that

$$
U_{1}(t, t-s) B^{1} \subset B^{1}, \quad \forall t \in \mathbb{R}, s \geq T_{B^{1}},
$$

and, therefore, the bounded set $B^{2} \subset C_{D(A), V}$ given by

$$
B^{2}=\bigcup_{t \in \mathbb{R}} \bigcup_{s \geq T_{B^{1}}} U_{1}(t, t-s) B^{1} \subset B^{1},
$$

is uniformly pullback absorbing for $U_{1}(\cdot, \cdot)$ in $C_{V, H}$.

If we finally prove that $B^{2}$ is a relatively compact subset of $C_{V, H}$, then, thanks to (20), Proposition 2.9 ensures that $\left\{B(t) \equiv \overline{B^{2}}\right\}_{t \in \mathbb{R}}$ is a family of compact subsets in $C_{V, H}$, which is also uniformly pullback attracting for $U(\cdot, \cdot)$, and the proof will be complete.

To prove the compactness of $\overline{B^{2}}$ we will use the Ascoli-Arzelà theorem. To this end, we need to check that $B^{2}$ is equicontinuous (i.e., $\forall \varepsilon>0, \exists \mu>0$, such that if $\left|\theta_{1}-\theta_{2}\right| \leq \mu$ then $\left\|v_{t}\left(\theta_{1} ; t-s, \phi\right)-v_{t}\left(\theta_{2} ; t-s, \phi\right)\right\|^{2}+\left|v_{t}^{\prime}\left(\theta_{1} ; t-s, \phi\right)-v_{t}^{\prime}\left(\theta_{2} ; t-s, \phi\right)\right| \leq \varepsilon$ for all $\phi \in D, t \in \mathbb{R}, s \geq$ 
$\left.T_{B^{1}}\right)$, and that for any $\theta \in[-r, 0]$ the set $\bigcup_{t \in \mathbb{R}} \bigcup_{s \geq T_{B^{1}}} \bigcup_{\phi \in B^{1}} v(t+\theta ; t-s, \phi)$ is precompact in $V$, and $\bigcup_{t \in \mathbb{R}} \bigcup_{s \geq T_{B}} \bigcup_{\phi \in B^{1}} v^{\prime}(t+\theta ; t-s, \phi)$ is precompact in $H$.

But, these two precompactness assertions hold, since both sets are bounded in $D(A)$ and $V$ respectively, and the injections $D(A) \subset V$, and $V \subset H$ are compact.

As for the equicontinuity, first notice that for any $\theta_{1}, \theta_{2} \in[-r, 0], \theta_{1}<\theta_{2}$,

$$
\begin{aligned}
\left\|v_{t}\left(\theta_{1} ; t-s, \phi\right)-v_{t}\left(\theta_{2} ; t-s, \phi\right)\right\| & \leq\left\|\int_{t+\theta_{1}}^{t+\theta_{2}} v^{\prime}(\sigma ; t-s, \phi) d \sigma\right\| \\
& \leq \int_{t+\theta_{1}}^{t+\theta_{2}}\left\|v^{\prime}(\sigma ; t-s, \phi)\right\| d \sigma \\
& \leq\left|\theta_{2}-\theta_{1}\right| \sup _{t+\theta_{1} \leq \sigma \leq t+\theta_{2}}\left\|v^{\prime}(\sigma ; t-s, \phi)\right\| \\
& \leq \rho_{1}\left|\theta_{2}-\theta_{1}\right| .
\end{aligned}
$$

Next,

$$
\begin{aligned}
& \left|v_{t}^{\prime}\left(\theta_{1} ; t-s, \phi\right)-v_{t}^{\prime}\left(\theta_{2} ; t-s, \phi\right)\right| \\
& \leq\left|\alpha \int_{t+\theta_{1}}^{t+\theta_{2}} v^{\prime}(\sigma ; t-s, \phi) d \sigma\right|+\left|\int_{t+\theta_{1}}^{t+\theta_{2}} A v(\sigma ; t-s, \phi) d \sigma\right|+\left|\int_{t+\theta_{1}}^{t+\theta_{2}} F(\sigma ; t-s, \phi) d \sigma\right| \\
& \leq \alpha \int_{t+\theta_{1}}^{t+\theta_{2}}\left|v^{\prime}(\sigma ; t-s, \phi)\right| d \sigma+\int_{t+\theta_{1}}^{t+\theta_{2}}|A v(\sigma ; t-s, \phi)| d \sigma+\int_{t+\theta_{1}}^{t+\theta_{2}}|F(\sigma ; t-s, \phi)| d \sigma \\
& \leq\left((\sigma+1) \rho_{1}+K_{7}\right)\left|\theta_{2}-\theta_{1}\right|,
\end{aligned}
$$

which is the required equicontinuity. The proof is now complete.

Remark 3.6. Although we have assumed in the previous section that the forcing term $f$ is autonomous and that the delay term satisfies some uniform (in time) global Lipschitz condition, it is possible to obtain, at least, the existence of the pullback attractor (non-uniform now) for our model. For instance, if we assume that $f \in L_{l o c}^{2}(\mathbb{R} ; H)$, and satisfies

$$
\int_{-\infty}^{t} e^{m s}|f(s)|^{2} d s<+\infty, \quad \text { for all } t \in \mathbb{R}, \quad \text { and } m>0,
$$

then, it is possible to perform our analysis so that one can now prove the existence of a family of compact attracting sets instead of a single compact set (see [3] for a similar discussion).

3.4. An application. Let us now show how our theory can be applied to the case in which the model contains some sort of variable delay.

Assume that operator $h$ is given by

$$
h\left(t, u_{t}\right)=\tilde{h}\left(u_{t}(-\omega(t))\right)-\beta \sin u_{t}(0)=\tilde{h}(u(t-\omega(t)))-\beta \sin u(t),
$$

with $\beta \in \mathbb{R}$, and $\tilde{h}: H \rightarrow H$ satisfying that $\tilde{h}(0)=0, \tilde{h} \in C^{1}(H ; H)$ where the Fréchet derivative $\delta \tilde{h}$ is globally bounded, and $\omega \in C^{1}(\mathbb{R}), \omega(t) \geq 0$ for all $t \in \mathbb{R}, r=\sup _{t \in \mathbb{R}} \omega(t) \in(0,+\infty)$, and $0<\omega_{*}=\sup _{t \in \mathbb{R}} \omega^{\prime}(t)<1$. Then, it is easy to check that $h$ satisfies assumptions (I)-(V) and, consequently, there exist the pullback and uniform forward attractors for our model. Observe that this situation corresponds to a model of Sine-Gordon type with delay forcing term. More 
precisely,

$$
\left\{\begin{aligned}
\frac{\partial^{2} u}{\partial t^{2}}+\alpha \frac{\partial u}{\partial t}-\Delta u+\beta \sin u & =f+\tilde{h}(u(t-\omega(t))), \quad t>\tau \\
u_{\mid \Gamma} & =0, \quad t \geq \tau-r \\
u(x, t) & =\phi(x, t-\tau), \quad x \in \Omega, t \in[\tau-r, \tau] \\
\frac{\partial u}{\partial t}(x, t) & =\frac{\partial \phi}{\partial t}(x, t-\tau), \quad x \in \Omega, t \in[\tau-r, \tau] .
\end{aligned}\right.
$$

\section{APPENDIX}

In our situation, i.e for $\left.H=L^{2}(\Omega), V=H_{0}^{1}(\Omega)\right)$ and $A=-\Delta$ with domain $H_{0}^{1}(\Omega) \cap H^{2}(\Omega)$, it follows that $A \in \mathcal{L}\left(V, V^{\prime}\right)$ is self adjoint and $\langle A u, v\rangle=((u, v))$.

Denote $I=(a, b)$. We then have the following result, which is a particular version of a more general one due to Strauss [17].

Theorem 3.7. Assume that $u \in L^{\infty}(I ; V) \cap W^{1, \infty}(I ; H)$ and $u^{\prime \prime}+A u \in L^{2}(I ; H)$. Then, $u \in C^{0}(\bar{I} ; V) \cap C^{1}(\bar{I} ; H)$ and

$$
\frac{d}{d t}\left(\langle A u, u\rangle+\left|u^{\prime}\right|^{2}\right)=2\left(u^{\prime \prime}+A u, u^{\prime}\right) \quad \text { in } \mathcal{D}^{\prime}(I) .
$$

As a straightforward consequence, we have the following result.

Theorem 3.8. If $u \in W^{1, \infty}(I ; V) \cap W^{2, \infty}(I ; H)$ and $u^{\prime \prime}+A u \in W^{1,2}(I ; H)$, then $u \in C^{0}(\bar{I} ; D(A)) \cap$ $C^{1}(\bar{I} ; V) \cap C^{2}(\bar{I} ; H)$, and $E q$. (26) holds.

Proof. It is obvious that $u \in C^{1}(\bar{I} ; V) \cap C^{2}(\bar{I} ; H)$. Moreover

$$
\left(u^{\prime \prime}+A u\right)^{\prime}=u^{\prime \prime \prime}+A u^{\prime}=\left(u^{\prime}\right)^{\prime \prime}+A u^{\prime} \in L^{2}(I ; H),
$$

with $u^{\prime} \in L^{\infty}(I ; V) \cap W^{1, \infty}(I ; H)$, and, by Theorem 3.7, $u^{\prime} \in C^{0}(\bar{I} ; V) \cap C^{1}(\bar{I} ; H)$.

The following result is crucial for our analysis.

Theorem 3.9. Suppose that $u \in L^{\infty}(I ; V) \cap W^{1, \infty}(I ; H)$ satisfies

$$
u^{\prime \prime}+\alpha u^{\prime}+A u=F \quad \text { in } \quad \mathcal{D}^{\prime}\left(I ; V^{\prime}\right),
$$

where $F \in L^{2}(I ; H)$ and $\alpha>0$. Then

(a) $u \in C^{0}(\bar{I} ; V) \cap C^{1}(\bar{I} ; H)$, and for any $\varepsilon \in \mathbb{R}$

$$
\begin{gathered}
\frac{d}{d t}\left(\|u\|^{2}+\left|u^{\prime}+\varepsilon u\right|^{2}\right)=2\left(F, u^{\prime}+\varepsilon u\right)-2 \varepsilon\|u\|^{2}+2(\varepsilon-\alpha)\left|u^{\prime}+\varepsilon u\right|^{2} \\
-2 \varepsilon(\varepsilon-\alpha)\left(u, u^{\prime}+\varepsilon u\right) \quad \text { in } \quad \mathcal{D}^{\prime}(I) .
\end{gathered}
$$

(b) Furthermore, if $F \in W^{1,2}(I ; H)$ and $u \in W^{1, \infty}(I ; V) \cap W^{2, \infty}(I ; H)$, then $u \in C^{0}(\bar{I} ; D(A)) \cap$ $C^{1}(\bar{I} ; V) \cap C^{2}(\bar{I} ; H)$, and

$$
\begin{aligned}
& \frac{d}{d t}\left(\left\|u^{\prime}+\frac{\alpha}{2} u\right\|^{2}+|A u-F|^{2}\right)+\frac{\alpha}{2}\left(\left\|u^{\prime}+\frac{\alpha}{2} u\right\|^{2}+|A u-F|^{2}\right) \\
& \leq \alpha|F|^{2}+\frac{4}{\alpha}\left|F^{\prime}\right|^{2}+\frac{\alpha^{3}}{8}\|u\|^{2} \quad \text { in } \quad \mathcal{D}^{\prime}(I) .
\end{aligned}
$$


Proof. (a) Fix $\varepsilon \in \mathbb{R}$, and denote $v=u^{\prime}+\varepsilon u$. We have

$$
\|u\|^{2}+|v|^{2}=|| u \|^{2}+\left|u^{\prime}\right|^{2}+2 \varepsilon\left(u^{\prime}, u\right)+\varepsilon^{2}|u|^{2},
$$

and from (26) it holds

$$
\begin{aligned}
\frac{d}{d t}\left(\|u\|^{2}+|v|^{2}\right)= & \frac{d}{d t}\left(\|u\|^{2}+\left|u^{\prime}\right|^{2}\right)+2 \varepsilon\left\langle u^{\prime \prime}, u\right\rangle+2 \varepsilon\left|u^{\prime}\right|^{2}+2 \varepsilon^{2}\left(u^{\prime}, u\right) \\
= & 2\left(F-\alpha u^{\prime}, u^{\prime}\right)+2 \varepsilon(F, u)-2 \varepsilon\langle A u, u\rangle-2 \varepsilon \alpha\left(u^{\prime}, u\right) \\
& +2 \varepsilon\left|u^{\prime}\right|^{2}+2 \varepsilon^{2}\left(u^{\prime}, u\right) \\
= & 2(F, v)+2(\varepsilon-\alpha)\left|u^{\prime}\right|^{2}+2 \varepsilon(\varepsilon-\alpha)\left(u^{\prime}, u\right)-2 \varepsilon\|u\|^{2} \\
= & 2(F, v)-2 \varepsilon\|u\|^{2}+2(\varepsilon-\alpha)\left(u^{\prime}, v\right) \\
= & 2(F, v)-2 \varepsilon\|u\|^{2}+2(\varepsilon-\alpha)|v|^{2}-2 \varepsilon(\varepsilon-\alpha)(u, v) .
\end{aligned}
$$

(b) Take $\varepsilon=\alpha / 2$, so that $v=u^{\prime}+\frac{\alpha}{2} u$. Then, $v \in C^{0}(\bar{I} ; V) \cap C^{1}(\bar{I} ; H)$ and

$$
\begin{aligned}
v^{\prime \prime}+A v & =\left(u^{\prime \prime}\right)^{\prime}+\frac{\alpha}{2} u^{\prime \prime}+A u^{\prime}+\frac{\alpha}{2} A u \\
& =F^{\prime}-A u^{\prime}-\alpha u^{\prime \prime}+\frac{\alpha}{2} u^{\prime \prime}+A u^{\prime}+\frac{\alpha}{2} A u \\
& =-\frac{\alpha}{2} u^{\prime \prime}+F^{\prime}+\frac{\alpha}{2} A u,
\end{aligned}
$$

and thus

$$
\frac{d}{d t}\left(\|v\|^{2}+\left|v^{\prime}\right|^{2}\right)=2\left(F^{\prime}, v^{\prime}\right)-\alpha\left(u^{\prime \prime}, v^{\prime}\right)+\alpha\left(A u, v^{\prime}\right) .
$$

On the other hand, as $v^{\prime}=u^{\prime \prime}+\frac{\alpha}{2} u^{\prime}=F-A u-\frac{\alpha}{2} u^{\prime}$, it follows

$$
\left|v^{\prime}\right|^{2}=|F-A u|^{2}+\frac{\alpha^{2}}{4}\left|u^{\prime}\right|^{2}-\alpha\left(F-A u, u^{\prime}\right) .
$$

Consequently,

$$
\frac{d}{d t}\left(\left|v^{\prime}\right|^{2}\right)=\frac{d}{d t}\left(|F-A u|^{2}\right)+\frac{\alpha^{2}}{2}\left(u^{\prime \prime}, u^{\prime}\right)-\alpha\left(F-A u, u^{\prime \prime}\right)-\alpha\left(F^{\prime}, u^{\prime}\right)+\alpha\left\|u^{\prime}\right\|^{2},
$$

whence,

$$
\frac{d}{d t}\left(\left|v^{\prime}\right|^{2}\right)=\frac{d}{d t}\left(|F-A u|^{2}\right)-\alpha\left(u^{\prime \prime}, u^{\prime \prime}+\frac{\alpha}{2} u^{\prime}\right)-\alpha\left(F^{\prime}, u^{\prime}\right)+\alpha\left\|u^{\prime}\right\|^{2} .
$$

From (30) and (31) we deduce, taking into account that $v^{\prime}=u^{\prime \prime}+\frac{\alpha}{2} u^{\prime}$,

$$
\frac{d}{d t}\left(\left|v^{\prime}\right|^{2}+|F-A u|^{2}\right)=2\left(F^{\prime}, u^{\prime \prime}+\alpha u^{\prime}\right)+\alpha\left(A u, u^{\prime \prime}+\frac{\alpha}{2} u^{\prime}\right)-\alpha\left\|u^{\prime}\right\|^{2} .
$$

Now, as $u^{\prime \prime}+\frac{\alpha}{2} u^{\prime}=F-A u-\frac{\alpha}{2} u^{\prime}$, we obtain from (32)

$$
\begin{aligned}
\frac{d}{d t}\left(\|v\|^{2}+|F-A u|^{2}\right)=2 & \left(F^{\prime}, F-A u\right)+\alpha(F, A u)-\alpha|A u|^{2} \\
& -\alpha\left(\left(v, \frac{\alpha}{2} u^{\prime}\right)\right)-\alpha\left(\left(u^{\prime}, u^{\prime}\right)\right) \\
= & 2\left(F^{\prime}, F-A u\right)+\alpha(F, A u)-\alpha|A u|^{2}-\alpha\left(\left(u^{\prime}, v\right)\right) \\
= & 2\left(F^{\prime}, F-A u\right)+\alpha(F, A u)-\alpha|A u|^{2}-\left.\alpha|| v\right|^{2} \\
& +\frac{\alpha^{2}}{2}((u, v))+\frac{\alpha}{2}|F|^{2}-\frac{\alpha}{2}|F|^{2},
\end{aligned}
$$


and, therefore,

$$
\begin{aligned}
& \frac{d}{d t}\left(\|v\|^{2}+|F-A u|^{2}\right)+\frac{\alpha}{2}\left(\|v\|^{2}+|F-A u|^{2}\right) \\
& =2\left(F^{\prime}, F-A u\right)-\frac{\alpha}{2}|A u|^{2}-\frac{\alpha}{2}\|v\|^{2}+\frac{\alpha^{2}}{2}((u, v))+\frac{\alpha}{2}|F|^{2} .
\end{aligned}
$$

Notice now that

$$
\begin{aligned}
2\left(F^{\prime}, F-A u\right) & =2\left(F^{\prime}, F\right)-2\left(F^{\prime}, A u\right) \\
& \leq \frac{2}{\alpha}\left|F^{\prime}\right|^{2}+\frac{\alpha}{2}|F|^{2}+\frac{2}{\alpha}\left|F^{\prime}\right|^{2}+\frac{\alpha}{2}|A u|^{2},
\end{aligned}
$$

and

$$
\frac{a^{2}}{2}((u, v)) \leq \frac{\alpha}{2}\|v\|^{2}+\frac{\alpha^{3}}{8}\|u\|^{2} .
$$

Then, thanks to (33)-(35), one can easily deduce (29).

\section{REFERENCES}

[1] T. Caraballo \& J.A. Langa, On the upper semicontinuity of cocycle attractors for non-autonomous and random dynamical systems, Dynamics of Continuous, Discrete and Impulsive Systems, Series A 10(2003), 491-513.

[2] T. Caraballo, P. Marín-Rubio \& J. Valero, Autonomous and non-autonomous attractors for differential equations with delays, J. Diff. Eqns. (2004) (to appear).

[3] T. Caraballo \& J. Real, Attractors for 2D-Navier-Stokes models with delays, J. Diff. Eqns. (2004) (to appear).

[4] D. Cheban, P.E. Kloeden \& B. Schmalfuss, The relationship between pullback, forwards and global attractors of nonautonomous dynamical systems, Nonlinear Dynamics $\&$ Systems Theory 2(2002), 9-28.

[5] V. Chepyzhov \& M. Vishik, Attractors for equations of mathematical physics, American Mathematical Society Colloquium Publications, 49. American Mathematical Society, Providence, RI, (2002).

[6] I.D. Chueshov, Introduction to the Theory of Infinite-Dimensional Dissipative Systems, Acta Scientific Publishing House, Kharkiv, Ukraine, (2002).

[7] H. Crauel \& F. Flandoli, Attractors for random dynamical systems, Probability Theory and Related Fields 100(1994), 365-393.

[8] H. Crauel, A. Debussche \& F. Flandoli, Random attractors, J. Dyn. Diff. Eq. 9(1995), No. 2, 307-341.

[9] J.K. Hale, Asymptotic Behavior of Dissipative Systems, Math. Surveys and Monographs, AMS, Providence, (1988).

[10] M.J. Garrido-Atienza and J. Real, Existence and uniqueness of solutions for delay evolution equations of second order in time, J. Math. Anal. Appl. 283(2003), 582-609.

[11] M.J. Garrido-Atienza and J. Real, Existence and uniqueness of solutions for delay stochastic evolution equations of second order in time, Stochastics and Dynamics 3(2003), 2, 141-167.

[12] P.E. Kloeden \& D.J. Stonier, Cocycle attractors in nonautonomously perturbed differential equations, $D y-$ namics of Continuous, Discrete and Impulsive Systems 4(1998), no. 2, 211-226.

[13] P.E. Kloeden \& B. Schmalfuss, Nonautonomous systems, cocycle attractorss and variable time-step discretization, Numer. Algorithms 14(1997), 141-152.

[14] J.L. Lions, Quelques méthodes de résolutions des probèmes aux limites non linéaires, Paris; Dunod, GauthierVillars, (1969).

[15] B. Schmalfuss, Backward cocycle and attractors of stochastic differential equations, in V. Reitmann, T. Redrich and N. J. Kosch (eds.), International Seminar on Applied Mathematics-Nonlinear Dynamics: Attractor Approximation and Global Behaviour (1992), 185-192.

[16] G. Sell, Non-autonomous differential equations and dynamical systems, Amer. Math. Soc. 127(1967), 241-283.

[17] W.A. Strauss, On continuity of functions with values in various Banach spaces, Pacific J. Math. 19(1966), No. 3, 543-551.

[18] R. Temam, Infinite Dimensional Dynamical Systems in Mechanics and Physics, Springer-Verlag, New York, (1988).

E-mail address, Tomás Caraballo: caraball@us.es

E-mail address, Peter E. Kloeden: kloeden@math.uni-frankfurt.de

E-mail address, José Real: jreal@us.es 
(Tomás Caraballo and José Real) Dpto. Ecuaciones Diferenciales y Análisis Numérico, Universidad de Sevilla, Apdo. de Correos 1160, 41080-Sevilla (Spain)

(Peter E. Kloeden) Fachbereich Mathematik, Johann Wolfgang Goethe-Universität, D-60054 Frankfurt am Main, Germany 